\title{
Beyond Imaging: Scanning Electron Microscope for the Quantitative Mass Measurement
}

\author{
Vladislav Krzyzanek ${ }^{1,2}$, Sebastian Tacke ${ }^{2}$, Kamila Dobranska ${ }^{1,3}$ and Rudolf Reichelt ${ }^{2}$ \\ 1. Institute of Scientific Instruments of ASCR, Brno, Czech Republic. \\ 2. University of Muenster, Institute of Medical Physics and Biophysics, Muenster, Germany. \\ 3. Brno University of Technology, Institute of Physical Engineering, Brno, Czech Republic.
}

Quantitative measurements of thin samples, e.g., mass determination of macromolecular assemblies, have been performed for many years by the dedicated scanning transmission electron microscopes (STEM) established at only a few institutions worldwide [1,2]. However, such quantitative measurements can also be performed by commercial high-resolution scanning electron microscopes (SEM) if they are appropriately extended. This includes mainly a very sensitive and well calibrated annular dark-field (ADF) detector, and a specific data processing, enabling to obtain simultaneously structural information and data on the mass thickness distribution.

In our experiments the cold field-emission SEM (S-5000, Hitachi Ltd.) was equipped with an ADF detector capable of single electron counting and a precise monitoring of the actual electron probe current $[3,4]$. This set-up allows quantitative imaging at low dose in order to minimize the electron beam induced artifacts. The quantitative studies utilize the transmitted scattered electrons combined with the Monte Carlo simulation of the scattering process MONCA [5]. The data analysis is performed using a highly automated data processing program MASDET [6] allowing calculations of different types of mass and various parameters, like the total mass of globular structures, the mass-per-length of filamentous structures, the mass-per-area of sheet-like structures, and local projected mass thickness.

For samples requiring cryo-preparation a controlled environment vitrification system was developed; rapidly frozen carbon coated grids containing the sample are mounted inside modified capsules for a cryo-holder (CT3500, Gatan Inc.). Up to nine grids can be subsequently freeze-dried in a vacuum chamber, and finally individual capsules containing the grids are transferred into the SEM specimen column and fixed to the cryo-holder CT3500 under defined conditions [7]. The imaging can be done in both room and low temperature.

The performance of the system was tested and verified with different materials including carbon, polystyrene and resin models with defined thickness, and with Tobacco Mosaic Virus (TMV) that is typically used as a calibration standard for measurements using the dedicated STEM [1,2]. Figure 1 shows mass measurement of TMV placed on a very thin C-film of a thickness $4 \mathrm{~nm}$. The ADF image (Figure 1a) was recorded at a dose of $\sim 300$ electrons $/ \mathrm{nm}^{2}$ (an electron probe current of $3 \mathrm{pA}$ ) and room temperature, and the resulting mass is shown on the Figure 1b. The mass was corrected according to the measured beam induced mass loss (Figure 1c).

The STEM imaging at lower electron energies recently became important for direct imaging of samples consisting of elements with lower atomic numbers without the need of any additional contrast enhancement like staining by heavy atoms. We show that ADF STEM can add a value to the common imaging like direct mass measurement without need of any calibration standards. Although this technique was originally developed for proteinaceous specimens, our extensions go beyond this and 
allow also for quantitative characterization of organic and inorganic specimens of any shape and composition. The measurable mass-thickness range is defined by the SEM and the detection geometry of the ADF detector. For our set-up the range is up to approximately the 7-fold amount of the electron tenmean free path and for protein complexes the mass range can be between tens of $\mathrm{kDa}$ and a few GDa. Presently, various applications are being tested, e.g., mass measurements of polyethylene hollow capsules, DNA-protein complexes, intermediate filaments.

\section{References:}

[1] JS Wall, Scanning Electron Microscopy 2 (1979), p. 291.

[2] A Engel, Ultramicroscopy 3 (1978), p. 273.

[3] V Krzyzanek and R Reichelt, Microscopy \& Microanalysis 13 (Suppl. 3) (2007), p. 80.

[4] H Nusse et al, Proc. Microscopy Conference MC2011, Kiel (2011).

[5] V Krzyzanek and R Reichelt, Microscopy \& Microanalysis 9 (Suppl. 3) (2003), p. 110.

[6] V Krzyzanek et al, Journal of Structural Biology 165 (2009), p. 78.

[7] S Tacke et al, Proceedings of the 15th European Microscopy Congress, Manchester 2012, Volume 3: Life Sciences (2012), p. 445.

[8] The authors acknowledge funding from the DFG, Grant Number RE 782/11, and from EC and MEYS CR, Grant Number CZ.1.07/2.3.00/20.0103.
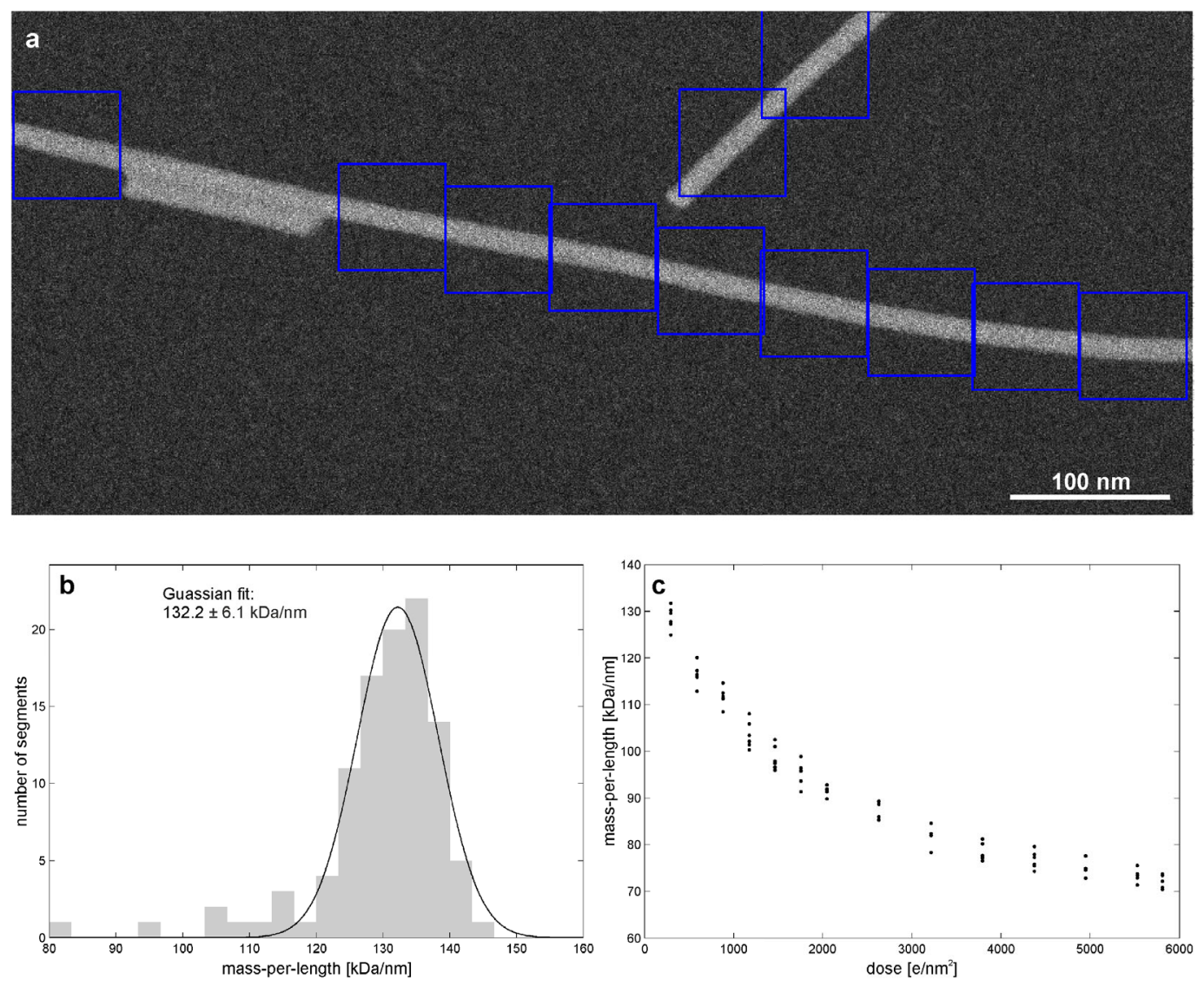

Figure 1. ADF micrograph of Tobacco mosaic virus acquired at $30 \mathrm{keV}$ (a), the mass distribution within 104 segments (b), and the mass loss dependence on the applied total electron dose for TMV at room temperature (c). 